\title{
On the Effects of an Installed Propeller Slipstream on Wing Aerodynamic Characteristics
}

\begin{abstract}
F. M. Catalano
This work presents an experimental study of the effect of an installed propeller slipstream on a wing boundary layer. The main objective was to analyse through wind tunnel experiments the effect of the propeller slipstream on the wing boundary layer characteristics such as: laminar flow extension and transition, laminar separation bubbles and reattachment and turbulent separation. Two propeller/wing configurations were studied: pusher and tractor. Experimental work was performed using two different models: a two-dimensional wing with a central cylindrical nacelle for the tractor configuration, and a simple two-dimensional wing with a downstream propeller for the pusher tests. The relative position between propeller and wing could be changed in the pusher model, and a total of 7 positions were analysed. For the tractor tests the relative propeller/wing was fixed, but three different propellers: two, three and four bladed were tested. Measurements included pressure distribution, hot wire anemometry and boundary layer characteristics by flow visualisation. The results showed that the pusher propeller inflow affects the wing characteristics by changing the lift, drag, and also delays the boundary layer transition and separation. These effects are highly dependent on the relative position of the wing/propeller. On the other hand, the tractor propeller slipstream induces transition and its effect is dependent on the number of blades.
\end{abstract}

Keywords: Wing /propeller interference, propeller slipstream, boundary layer.

\section{Introduction}

During the 1980s a large number of works [1-4] on UDF (unducted fans) or propfans brought attention back to the use of advanced propellers in transport aviation. All these works pointed to the potential benefits in fuel efficiency and $\mathrm{T} / \mathrm{O}$ thrust of the new propellers. Despite the fact that attention on propofans has decreased, there is still great interest in the use of propellers in general aviation and commuter $[2,4,8]$ aircraft as well as in RPV and unmanned aircraft [5]. For these classes of aeroplane the distance between the wing and the propeller can be close enough to induce quite large effects on the wing surfaces, especially when the propellers are operating at high thrust as in take-off and climb. At take-off the aircraft speed is close to stall velocity and the whole process from rotation to climb-out involves a large range of incidence with the propeller operating all the time at maximum thrust. Therefore, the effect of the propeller inflow on the wing in this situation can be of considerable magnitude [6,9]. Since the 1930s a large number of investigations have been performed on the effects of the slipstream on a wing and/or other components of the aircraft, including tractor and pusher configurations [13]. Almost a half of these works have been on steady loads or steady state wing/propeller interaction. Optimisation analyses performed by Kroo [10] and Miranda [11] have demonstrated that propeller/wing interaction, for the case of the tractor configuration, can result in significant wing drag reduction. Recent work [5, 6] and also demonstrated that laminar flow could be increased when pusher propellers are installed in convenient positions behind a wing, resulting in less friction drag. Concerning aircraft drag reduction we have to take into account the effect of the propeller slipstream, (here the propeller inflow is also considered as slipstream), on the wing boundary layer characteristics. Tractor and pusher propellers affect the boundary layer of a wing in completely different way. The tractor propeller acts in a unsteady fashion, due to the propeller wake and tip vortex crossing the wing surfaces. Such an effect can promote transition [12] or induce an alternation between laminar and turbulent states. On the other hand, a pusher propeller only affects the flow angularities on the wing surfaces, and for some positions it can alleviate the adverse pressure gradient and so prevent separation or/and increase laminar flow. This paper describes two experimental approaches to the analysis of the problem of wing/propeller interference. The first set of experiments was designed to analyse the effect of three different tractor propellers on the wing boundary layer. It was decided to use propellers with two, three and four blades in order to investigate the effect of the propeller wake and tip vortex frequency crossing the wing The second method concentrated on testing the effect of a high thrust pusher propeller driven by a hydraulic motor on a two-dimensional wing at a wide range of incidence and with the propeller also positioned at several positions behind the wing. Measurements included pressure distributions for the pusher case only, flow visualisation for both cases and hot wire measurements for the tractor case.

\section{Experimental set up}

\section{Pusher set up:}

A Wortmann FX63-137 profile wing with a chord of $0.34 \mathrm{~m}$ was used for the tests. The wing carried 82 pressure tappings around the centre line chord. A $0.52 \mathrm{~m}$ diameter three blade propeller driven by a $20 \mathrm{hp}$ hydraulic motor was used. For the pressure measurements an $8 \mathrm{ft} \times 4 \mathrm{ft}$ open return low speed wind tunnel was used with the wing positioned vertically in the working section (Fig. 1). The propeller was mounted on a separated pylon, which could be moved in order to set the propeller/wing positions. The wing could be moved vertically through the working section in order to measure the spanwise effect of the propeller on the surface pressure distribution. 


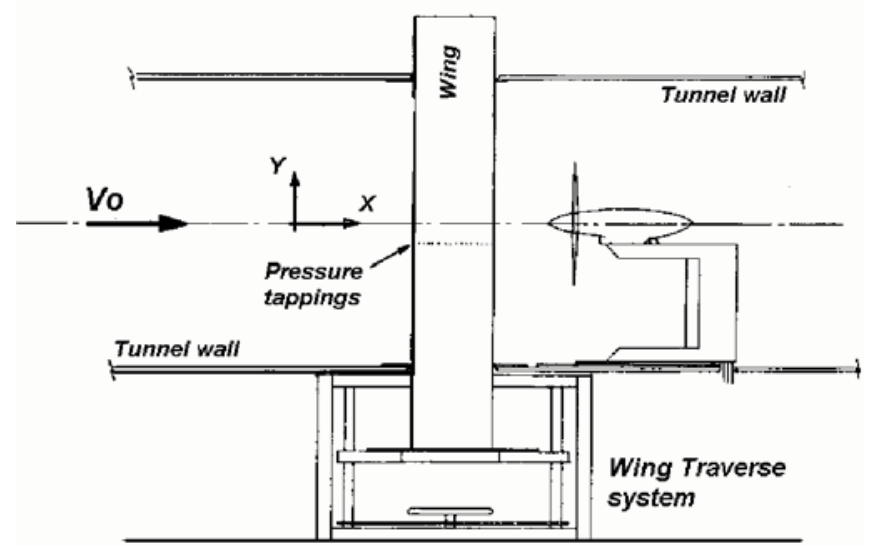

Fig. 1: Pressure measurements, pusher case

The force and moment measurements were made in an $8 \mathrm{ft} \times 6 \mathrm{ft}$ closed-circuit low speed wind tunnel using a similar arrangement for the propeller to that described above. The wing was attached vertically to a six-component balance and spanned the tunnel except for a $3 \mathrm{~mm}$ gap at one end so that force measurements could be made (Fig. 2). Flow visualisation was carried out using both sublimation and oil

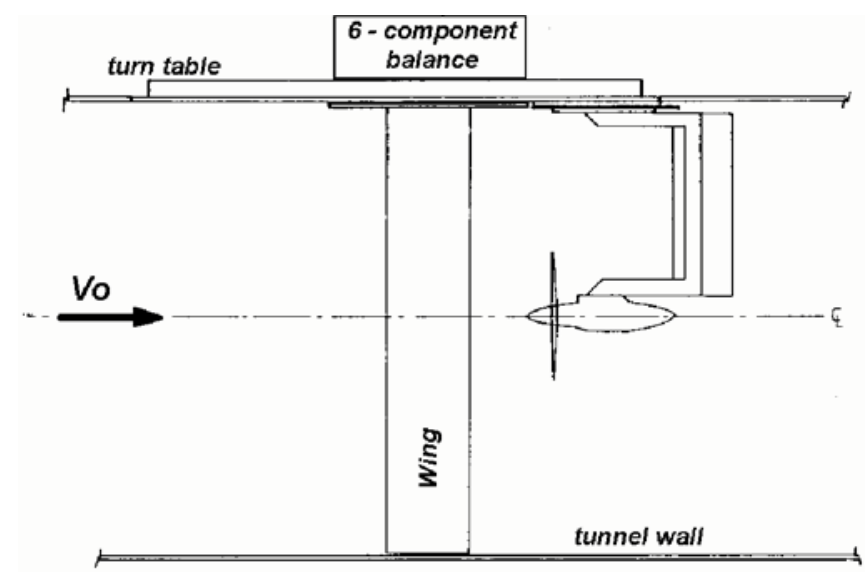

Fig. 2: Force and moment test set-up for the pusher case

technique. The seven wing/propeller position (Table 1) was tested through the incidence range of -4 to +20 degrees with and without a trip wire. Wing surface pressure distribution was measured at 10 spanwise positions. The Reynolds number was set at 0.45 millions and the propeller was run at a thrust coefficient of $C_{T}=0.15$ with an advance ratio of $J=0.33$. These propeller characteristics were chosen in order to simulate a high power condition such as take-off and climb.

\section{Tractor set-up:}

The same Wortmann FX63-137 profile was used to construct two wings with a chord of $0.28 \mathrm{~m}$. These two wings were Table 1 Propeller Positions for the pusher case

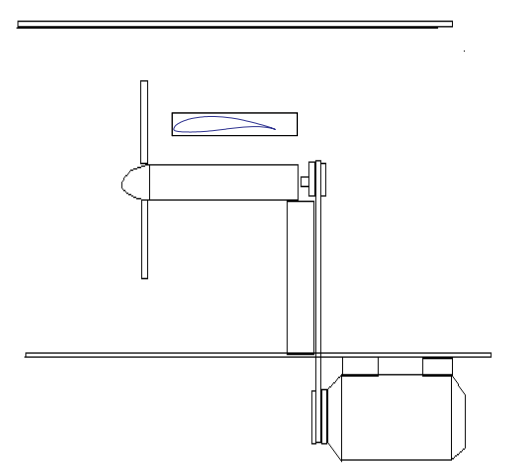

Fig. 3: Tractor propeller set-up

attached to a nacelle of cylindrical shape, Fig. 3 and 4. Inside the nacelle there was a shaft and two ball bearings, with the propeller and pulley attached to each end. The propeller was driven by a 5 Hp electric motor through a 1:2 pulley/belt system. A frequency inverter controlled the Motor/Propeller speed. The model span was $1 \mathrm{~m}$ long and the nacelle diameter $0.9 \mathrm{~m}$. The wing tips were positioned close to the wind tunnel wall in order to keep tip vortex at a minimum. Fig. 4 shows the

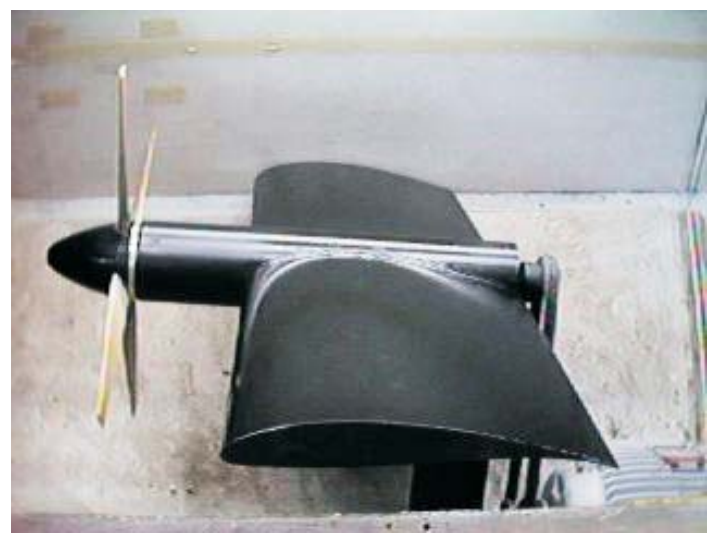

Fig. 4: Tractor propeller model

tractor propeller model mounted inside the wind tunnel working section. The wind tunnel is of the open circuit type with a $1 \mathrm{~m} \times 1 \mathrm{~m}$ working section. The three and four blade propellers had the same diameter of $0.40 \mathrm{~m}$ and the two-blade propeller $0.36 \mathrm{~m}$.

All experimental tests performed with the tractor model were conducted at a Reynolds number of 350.000 with the wing without any transition trip. The propeller speed was $7.000 \mathrm{rpm}$, resulting in an average advance ratio of $J=0.43$ for the three and four bladed propellers, and $J=0.48$ for the two bladed propeller. The visualisation technique used for transition localisation was by sublimation that consisted of spraying naphthalene diluted in a volatile solvent on the wing surfaces. Also oil flow visualisation was used for determining the wing surface characteristics such as laminar bubble sepa-

\begin{tabular}{|l|c|c|c|c|c|c|c|}
\hline Propeller position & POS 01 & POS 02 & POS 03 & POS 04 & POS 05 & POS 06 & POS 07 \\
\hline Distance From TE & $0.5 \mathrm{C}$ & $0.5 \mathrm{C}$ & $0.5 \mathrm{C}$ & $0.85 \mathrm{C}$ & $0.85 \mathrm{C}$ & $0.85 \mathrm{C}$ & $0.5 \mathrm{C}$ \\
\hline Above + below - chord & 0.0 & $0.23 \mathrm{C}$ & $0.46 \mathrm{C}$ & $0.46 \mathrm{C}$ & $0.23 \mathrm{C}$ & 0.0 & $0.19 \mathrm{C}(-)$ \\
\hline
\end{tabular}



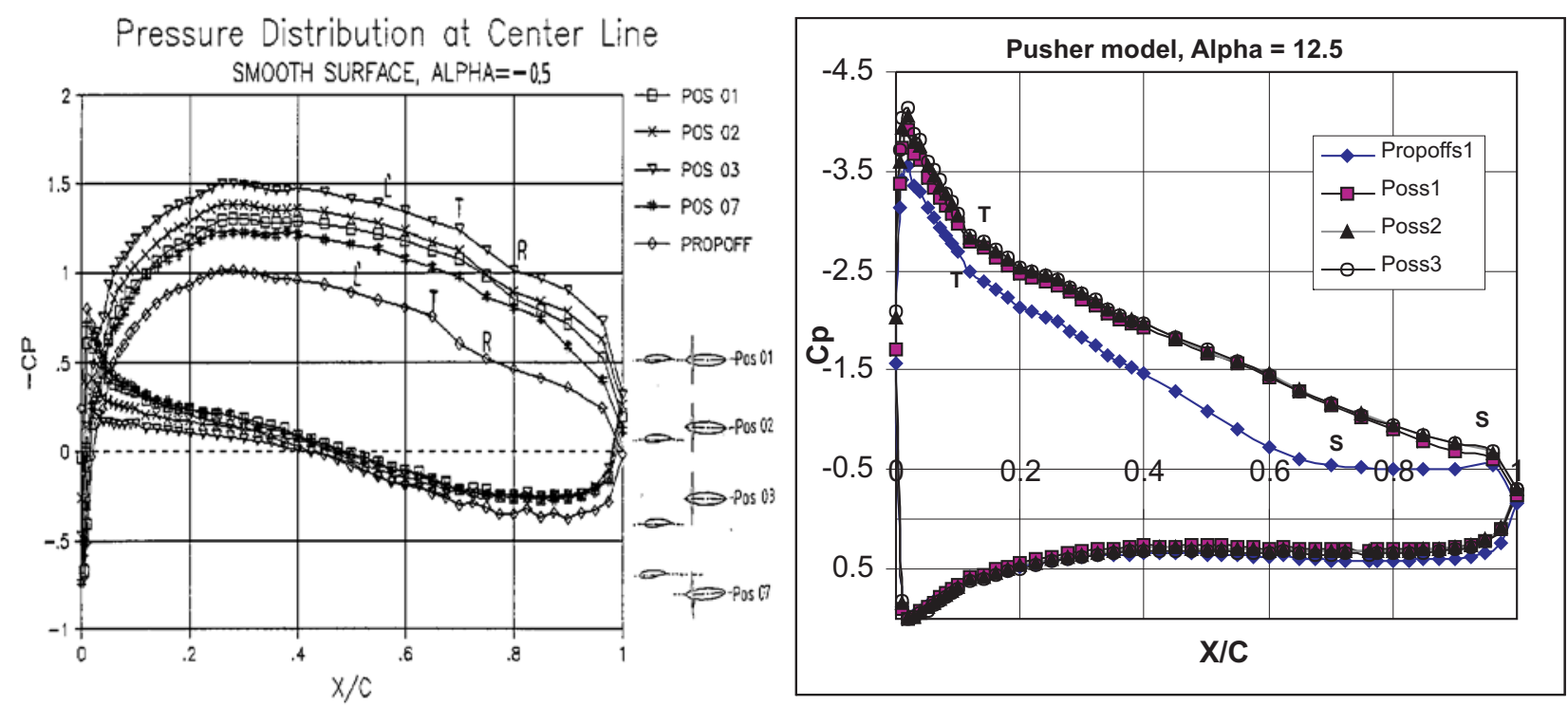

Fig. 5: Pressure distribution at the centre line of the pusher model wing

ration, turbulent separation, etc. Hot wire anomometry was carried out at the $40 \%$ chord position of the upper surface of the wing in 30 spanwise positions. The hot wire was kept at $1 \mathrm{~mm}$ from the wing surface in order to ensure that the measurements were taken inside the boundary layer for the whole probe traverse. A constant temperature hot wire anemometer with a traverse gear was used in the experiments.

\section{Results}

\section{Pusher Propeller model:}

The increase in suction on the upper surface of the wing due to the propeller is clearly shown in the pressure distributions of Fig. 5, which resulted in a gain in $C_{L}$ as shown in the $C_{L}-\alpha$ curve of Fig. 6 . The effect of the propeller is larger at the working incidence angles ( -4 to 6 degrees) for propeller positions above the wing's chord line and close to the trailing edge due to the increase in effective incidence and camber in-

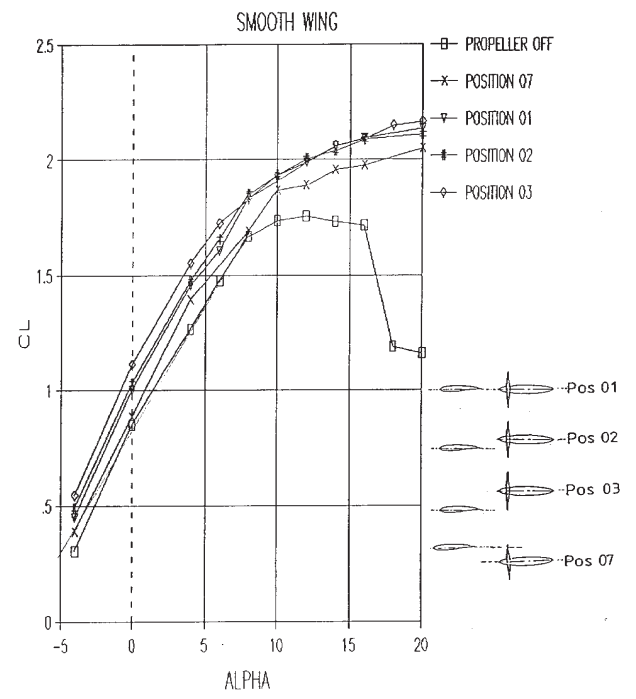

Fig. 6: $C_{L^{-}} \alpha$ for the pusher model wing

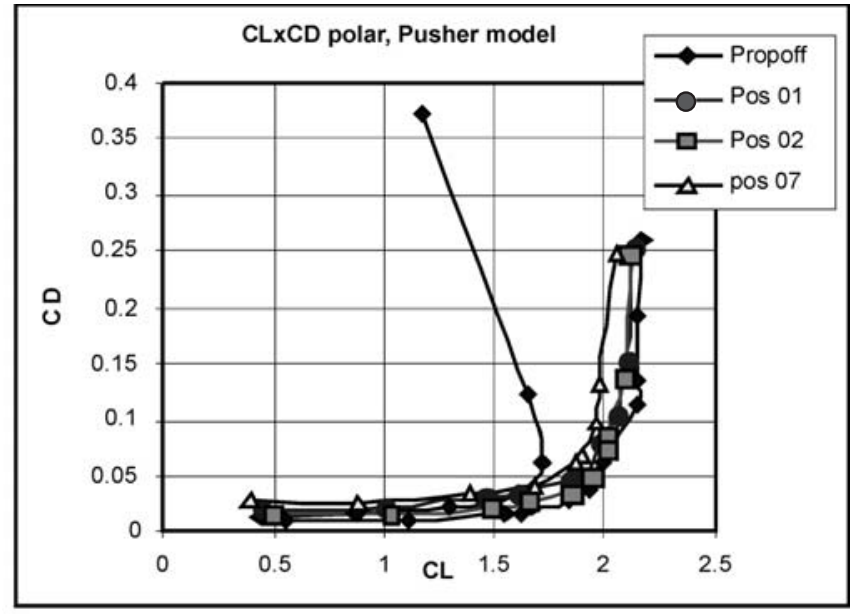

Fig. 7: Drag polar for pusher model

fluenced by the propeller inflow. A direct consequence of this increase of suction on the upper surface of the wing is an increase of pressure drag, as shown in the $C_{L}-C_{D}$ curve of Fig. 7. At high incidence angles part of this gain in $C_{L}$ is due to a delay in turbulent separation, as demonstrated by the movement downstream of the separation point $S$ in the $\alpha=12.5^{\circ}$ curve of Fig. 5. Flow visualisation using a smoke stream also showed the effect of the propeller on separation and upwash angle, as can be seen in Fig. 8. Because the boundary layer transition, in this case, is free from any trip, and also due to the low Reynolds number of the experiment, the effect of the propeller on changing local flow incidence affects the transition front. This effect can be seen in Figs. 9 and 10 by the movement of the transition front (determined by sublimation). The maximum effect on the transition front occurs at the centre of the wing and also acts on the laminar separation bubble, as can be seen in Fig. 5 with a change in the position of the point laminar separation $(L)$ and reattachment $(R)$ This effect decreases after incidence angles greater than 8 degrees and may even promote transition, as the effect of the propeller inflow at the leading edge is an increase of upwash. This 

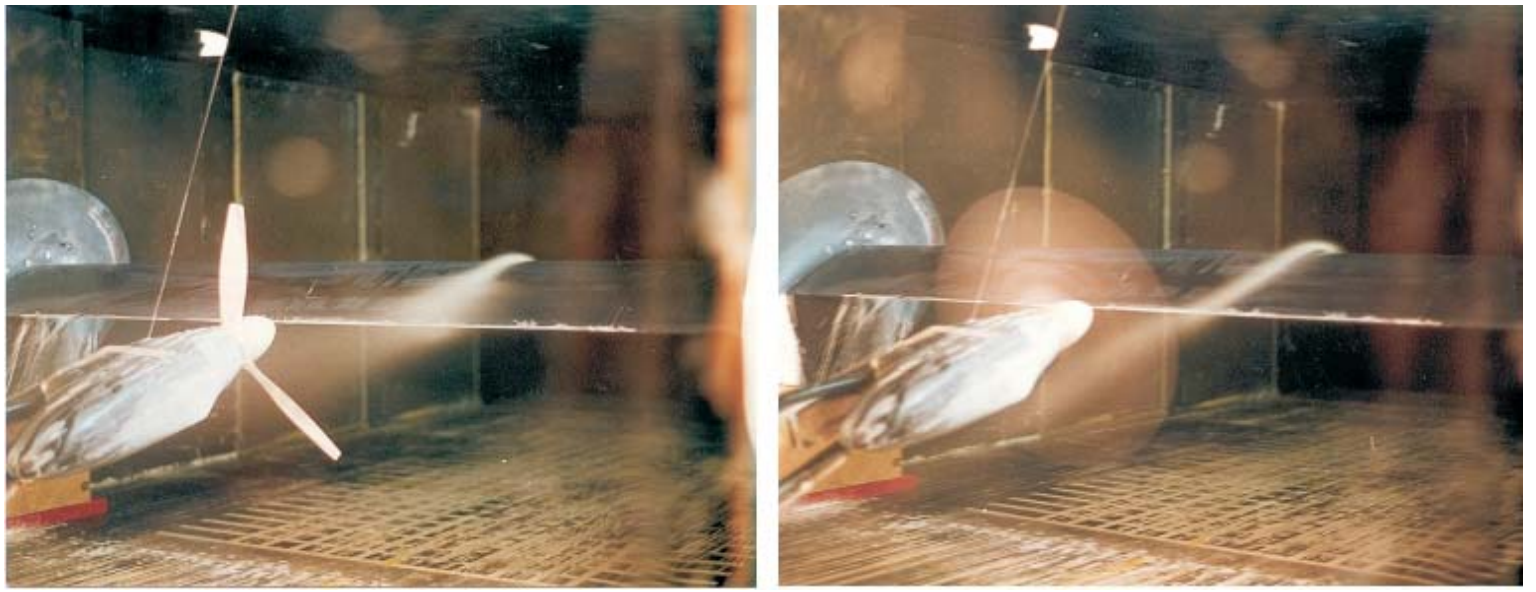

Fig. 8: Effect of the pusher propeller on separation, $\alpha=14^{\circ}$

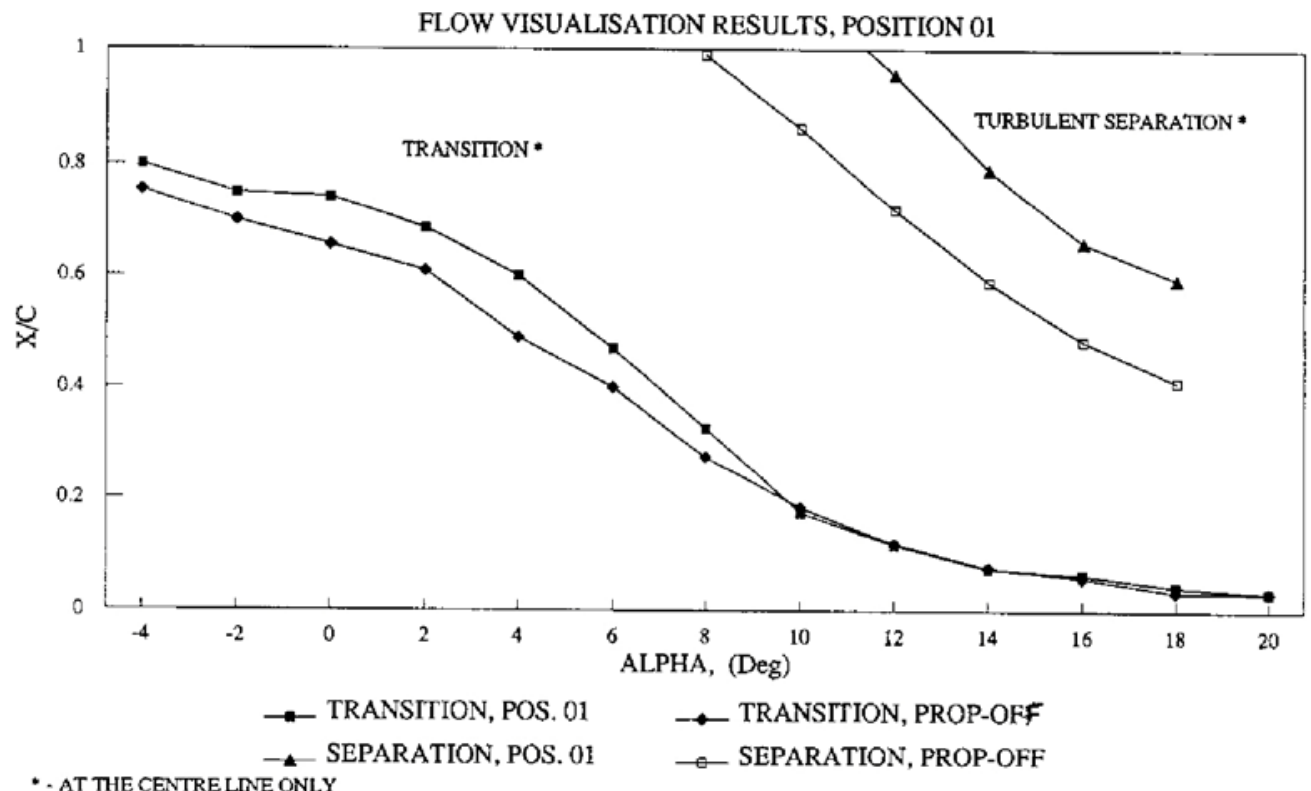

Fig. 9: Transition and separation points at centre line for pusher model at position 01

effect for two incidence angles can be seen in Fig. 11, which also shows that for high incidence angles, near the leading edge, the flow incidence induced by the propeller can move the transition front forward. This phenomenon is especially

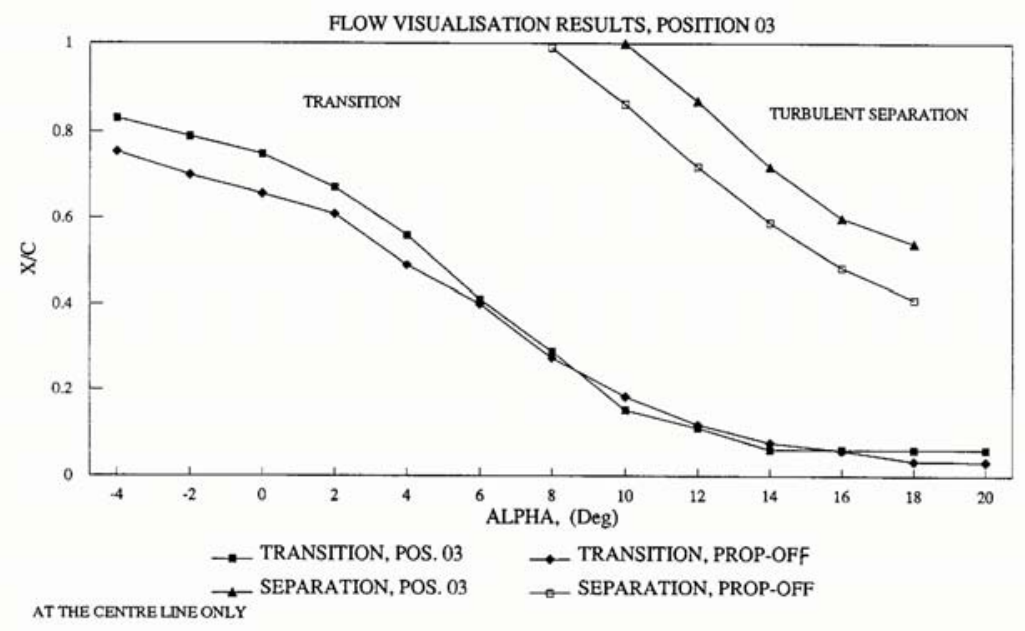

Fig. 10: Transition and separation points at the centre line for pusher model, position 03 


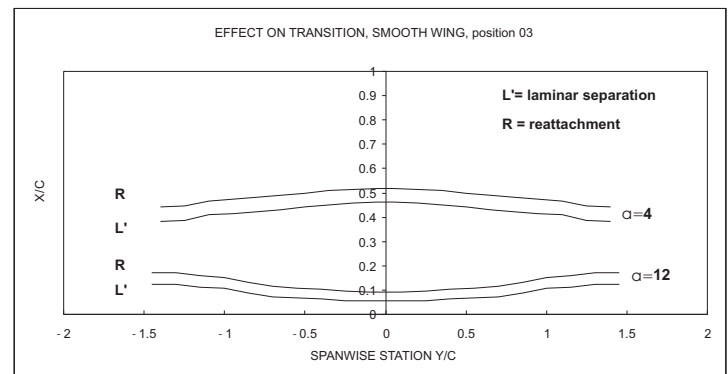

Fig. 11: Effect on spanwise transition due to the pusher propeller at position 03

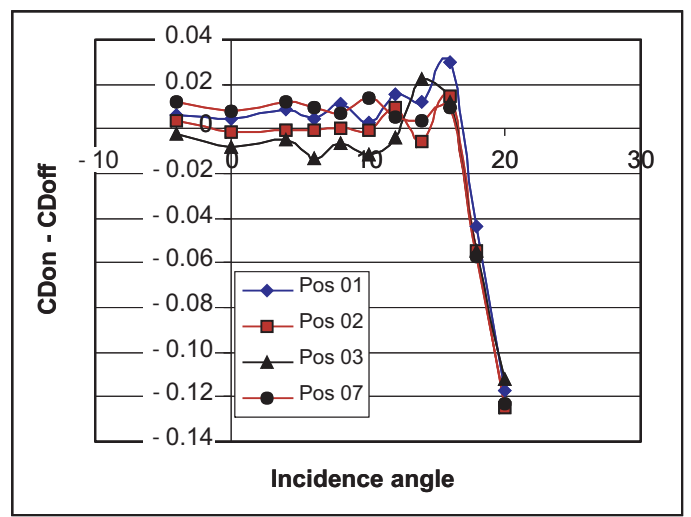

Fig. 12: Variation of CD for the pusher propeller on, compared with the propeller off case

intense for propeller positions above the chord line, but even so it is much less intense than the backward movement of the turbulent separation front due to the propeller inflow. Therefore, the gain in friction drag due to the increase of laminar flow found in low incidence angles is compensated by the increase of pressure drag and, on the other hand, for high incidence angles the decrease in pressure drag due to the delay of turbulent separation is the main benefit. These results can be seen in Fig. 12, which also shows that for propeller position 03 there is a decrease in drag for the working range of incidence angles. This happens not only due to the extended laminar flow but mainly due to a shift forward of the resultant force which will thus produce a small thrust force.

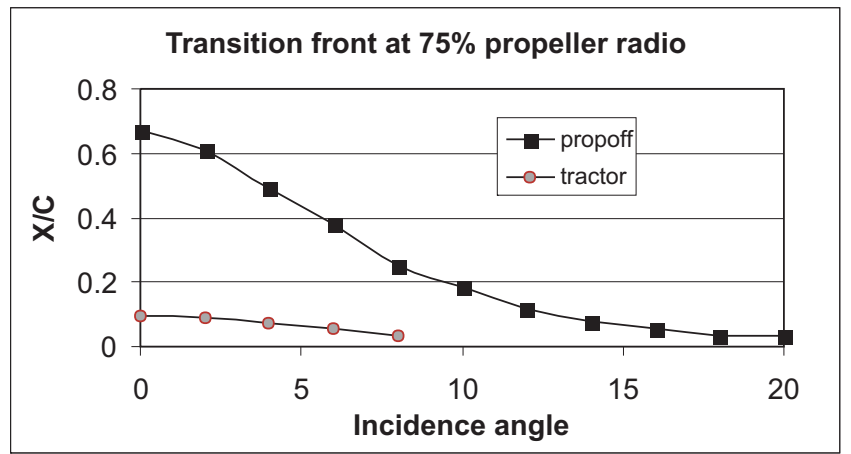

Fig. 13: Transition front for the tractor model, from flow visualisation

\section{Tractor Propeller Model:}

Due to working section restrictions, the range of incidence angles was limited from $0^{\circ}$ to $8^{\circ}$. In this phase of the tests, only flow visualisation and hot wire measurements were carried out. The first series of tests were to analyse the transition front using the sublimation technique. Naphthalene was sprayed only on the upper surface. The results are plotted in Fig. 13 for the three propellers and the measurement was taken in a spanwise station correspondent to $75 \%$ of the propeller radius. The results showed that inside the slipstream the transition front was brought close to the leading edge. It was found that there is no measurable difference between the effects of the three propellers, at least with the sublimation technique. Also it was not possible to observe if there was any difference between the left and right wing flow due to the propeller wake swirl. Fig. 14 shows a sketch of the transition front on the upper surface wing. Flow visualisation using the oil flow technique was more elucidating because it showed better the flow pattern of the wing. Fig. 14 shows the whole left wing at $4^{\circ}$ with the different oil flow patterns. It can be seen that the laminar separation bubble was washed out inside the slipstream and that the effect of the slipstream extends further than the propeller radius due to the viscous mixing between slipstream and external flow. Hot wire measurements were effective in order to find the effect of the blade wake crossing frequency. Fig. 15 shows the time history of the velocity inside the boundary layer for the two-bladed propeller. The periodic effect of the blade wake crossing the boundary layer can be seen. Figs. 16 and 17 show the time history of velocity for the
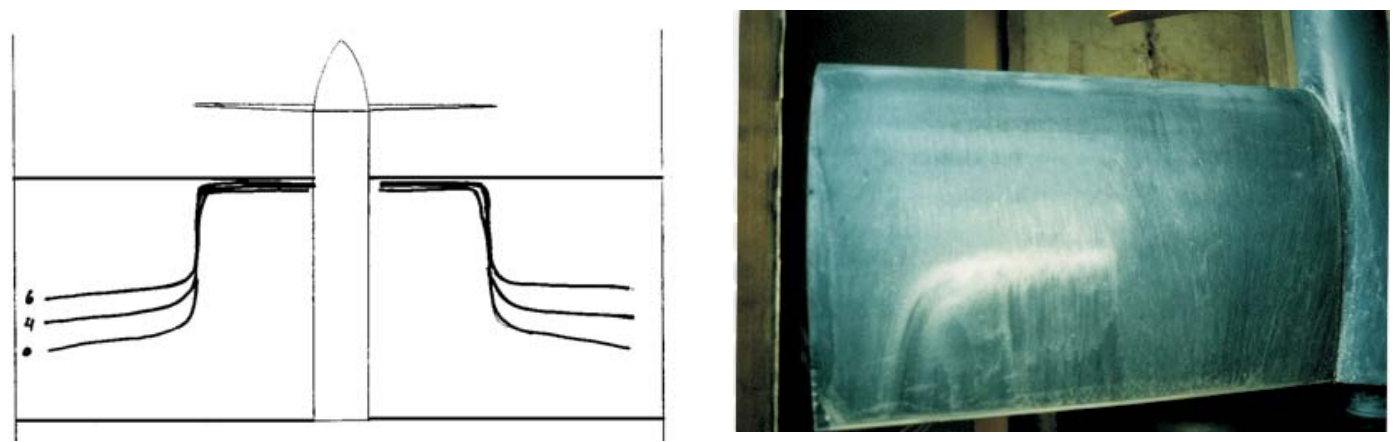

Fig. 14: Location of the transition front determined by flow visualization 


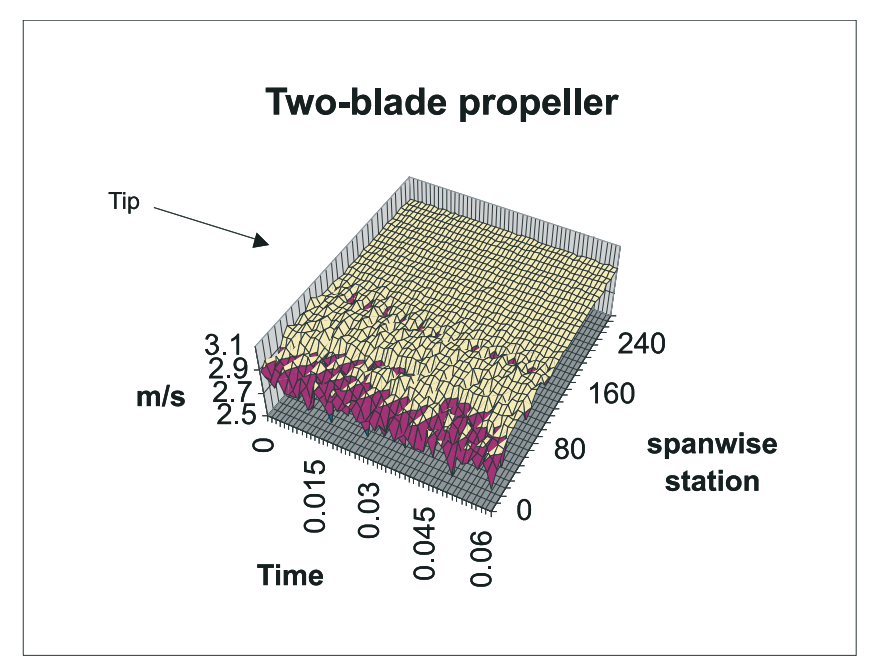

Fig. 15: Time history of the velocity fluctuations inside the tractor model boundary layer for the two-bladed propeller

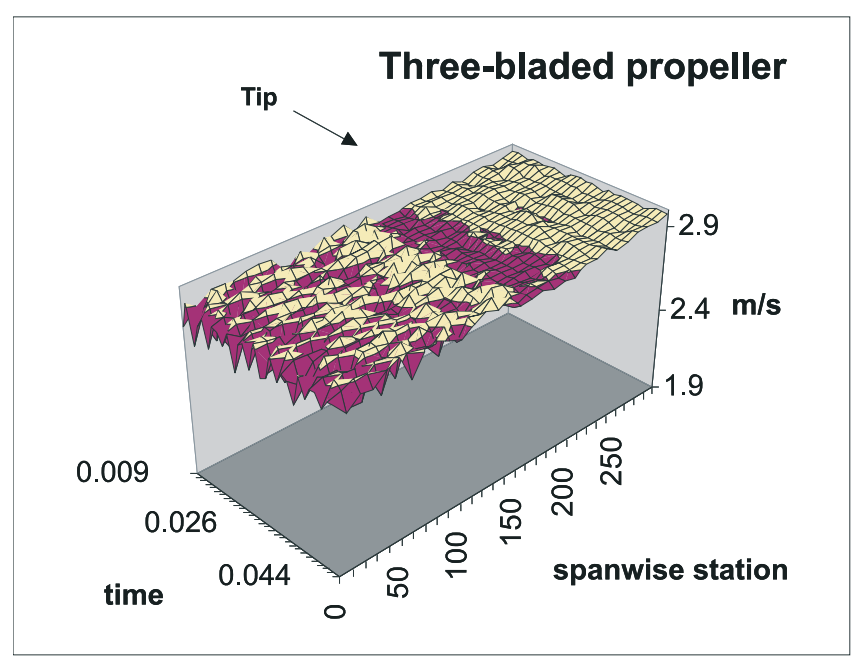

Fig. 16: Time history of the velocity fluctuations inside the tractor model boundary layer for the three-bladed propeller

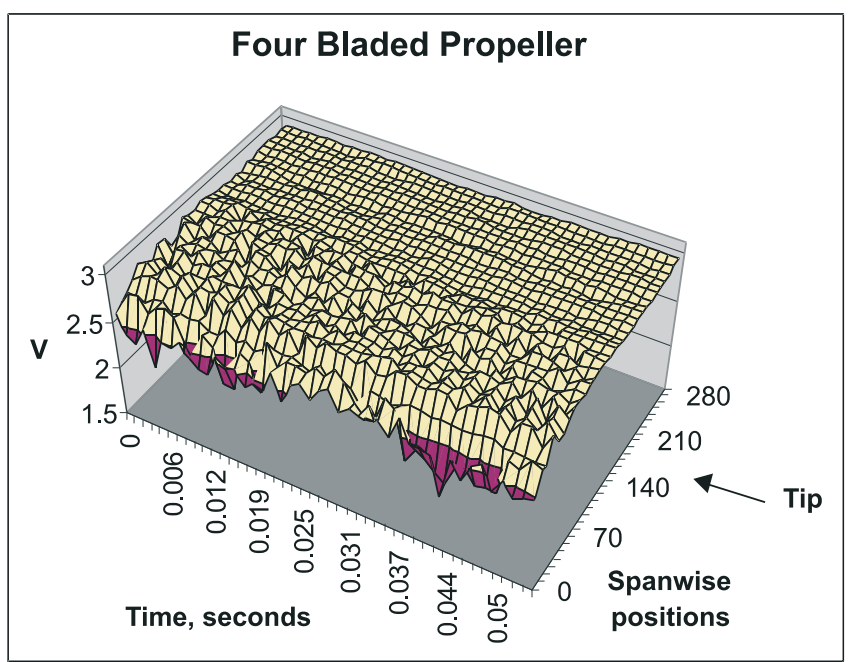

Fig. 17: Time history of velocity fluctuations for the four-bladed propeller

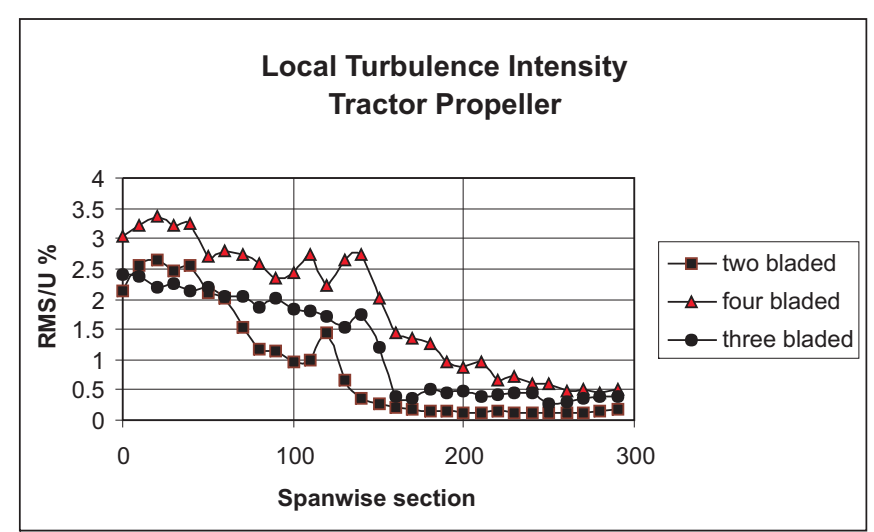

Fig. 18: Spanwise turbulence intensity

three and four bladed propellers. It can be observed from these figures that when increasing the frequency in the wake that is passing over the wing the injection of turbulence to the boundary layer from the blade wakes is much more intense. The turbulence intensity for the three propellers is plotted in Fig. 18.

\section{Conclusions}

The effect of a pusher and a tractor propeller on the flow over a straight wing was investigated by wind tunnel tests. A total of 7 different configurations of the pusher model were investigated and three different propellers were used for the tractor model. The propeller induced flow over the wing surfaces, thus increasing lift, pressure drag, and delaying turbulent separation. For the pusher propeller the effect was more intense on the rear of the wing but can also extend to the front by changing the upwash angle. The propeller effects are very dependent on the relative propeller/wing position. Over the working range of incidence angles, pusher propeller positions above the wing gave the best results. The propeller inflow can also delay transition by preserving laminar flow on a smooth wing at low Reynolds number due to the alleviation of the adverse pressure gradient at the rear of the wing. For the tractor propeller it was found that the slipstream passing over the wing promotes transition, changing its position to near the leading edge. If a laminar flow wing or a low Reynolds profile is used inside the slipstream, laminar flow can decrease $80 \%$ of that for a clear wing with no propeller flow. Also if a multi-blade propeller is in use it can destroy the intermittent shift of laminar to turbulent flow encountered when a two-blade propeller wake passes over a laminar wing as pointed out by Howard et al [12]. Pusher propeller wing-body configurations are still attractive when compared with the tractor configuration, particularly concerning wing flow and cabin noise.

\section{References}

[1] Henne P. A., Dahlin J. A., Peavey C. C., Gerren D. S.: "Configuration design Studies and Wind Tunnel Tests of an Energy Efficient Transport with a High Aspect Ratio Supercritical Wing”. NASA CR-3224, May, 1982. 
[2] Goldsmith I. M.: "A Study to Define the research and Technology Requirements for Advanced Turbo/Propfan Transport Aircraft". NASA CR-166138, 1981.

[3] Dunham D. M., Gentry G. L. Jr., Gregory M. S., Applin Z. T., Quinto P. F.: "Low Speed Aerodynamic Characteristics of an Twin-Engine General Aviation Configuration with Aft-Fuselage-Mounted Pusher Propellers". NASA TP-2763, 1987.

[4] Coe P. L. Jr., Turner S. G., Owens D. B.: "Low Speed Wind Tunnel Investigation of the Flight Dynamics Characteristics of an Advanced Turboprop Business/Commuter Aircraft Configuration”. NASA TP-2982, 1990.

[5] Catalano, F. M., Stollery J. L.: "The Effect of a High Thrust Pusher Propeller on the Aerodynamic Characteristics of a Wing at Low Reynolds". ICAS - 94-6.1.3, Anaheim (California, USA) September 1994.

[6] Catalano F. M., Maunsell M. G.: "Experimental and Numerical Analysis of the effect of a pusher propeller on a wing and Body". $35^{\text {th }}$ AIAA Aerospace Sciences Meeting and Exhibit, Reno (NV, USA), January 6-10, 1997.

[7] Maunsell, M. G.: "A Study of Propeller/wing /body Interference for a Low Speed Twin-Engined Pusher Configuration”. ICAS-90-5.4.3, 1990.

[8] Johnson J. L., White E. R.: "Exploratory Low Speed Wind Tunnel Investigation of an Advanced Commuter
Configuration Including an over-the-wing Propeller Design". AIAA-83-2531, 1983.

[9] Witkowski D. P., Johnston R. T., Sullivan J. P.: "Propeller/Wing Interaction”. 27 $7^{\text {th }}$ Aerospace Science Meeting Reno (NV, USA), January 9-12, 1989.

[10] Kroo I.: "Propeller-Wing Interaction for Minimum Induced Loss". AIAA 84-2470, 1984.

[11] Miranda L. R., Brennan J. E.: "Aerodynamics Effects of Wing Tip Mounted Propellers and Turbines”. AIAA 86-1802, 1986.

[12] Howard R. M., Milley S. J., Holmes B. J.: “An Investigation of the Propeller Slipstream on a laminar Wing Boundary Layer". SAE Paper No. 850859, 1985.

[13] Thompson J. S., Smelt R., Davison B., Smith F.: “Comparison of Pusher and Tractor Propeller Mounted on a Wing". Reports and Memoranda R\&M, No. 2516 June 1940 .

Fernando M. Catalano M.Sc Ph.D, MRAeS

Aircraft Laboratory

University of Sao Paulo - Brazil

Ave do Trabalhador SaoCarlense 400

Cep 13566-590 Sao Carlos SP 